\title{
NANO-ENCAPSULATION OF Lippia origanoides KUNTH. ESSENTIAL OIL BY CHITOSAN-CAFFEIC ACID NANOGEL
}

\author{
Elisa T. S. Damasceno ${ }^{a}$, Regiamara R. Almeida ${ }^{a}$, Stephanne Y. B. de Carvalho ${ }^{a}$, Sara S. Vieira ${ }^{\mathrm{b}}$, Valdir Mano and Luiz \\ Gustavo de L. Guimarães ${ }^{a, *,(1)}$ \\ ${ }^{a}$ Departamento de Ciências Naturais, Universidade Federal de São João del-Rei, 36301160 São João del-Rei - MG, Brasil \\ bDepartamento de Química, Universidade Federal de Minas Gerais, 31270-901, Belo Horizonte - MG, Brasil
}

Recebido em 06/06/2019; aceito em 20/08/2019; publicado em 27/11/2019

\begin{abstract}
In this study, a nanogel was developed from a natural polymer, chitosan, to be used in the Lippia origanoides essential oil encapsulation. The encapsulation process was used in order to improve the functional capacity of this essential oil as an antioxidant. To obtain the nanogel, the chitosan was modified with caffeic acid using EDC coupling agent. The obtained material was characterized by FTIR and TGA, revealing the formation of the chitosan-caffeic acid bond. The size, morphology and essential oil amount incorporated to the nanogel were investigated by DLS, SEM and gravimetric analysis, respectively. SEM and gravimetric analysis indicated that oil were efficiently trapped inside the CS-CA matrix, with encapsulation efficiency of $42 \pm 2 \%$. The EDL studies showed that the encapsulation of essential oil in the nanogel significantly affected your structure, with a average particle size of $417.26 \pm 55.96$. After essential oil incorporation into the CS-CA nanogel it was also observed a increase in its antioxidant capacity in terms of elimination of DPPH and ABTS radicals, and ferric reducing antioxidant power (FRAP). The successful development of this CS-CA nanogel loaded with L. origanoides essential oil will lead to a better applicability of this essential oil as an effective antioxidant.
\end{abstract}

Keywords: Nanoparticles; natural polymer; encapsulation; thymol; antioxidant activity.

\section{INTRODUCTION}

Natural products represent one of the most important sources of available medicines. In the latest years, a large portion of the new drugs allowed have been developed directly from natural products as well as produced by synthetic modifications. ${ }^{1}$ Plant species produce important secondary metabolites, including essential oils. These are recognized to play an important role in potent biological activities, including antibacterial, antifungal and antioxidant. ${ }^{2}$

Many of the constituents present in essential oils, including thymol and carvacrol monoterpenes, and their $\gamma$-terpinene and p-cymene biosynthetic precursors, contribute significantly to their biological activities. ${ }^{3}$ Several constituents of the essential oils are able to neutralize the free radicals, turning them promising in the research and development of new antioxidant agents. ${ }^{4}$ Recently, the essential oil of Lippia origanoides Kunth has attracted attention because of its considerable potential as an antioxidant. ${ }^{5,6}$

The antioxidant compounds are characterized by their ability to retard oxidative reactions caused by free radicals excess. These compounds are effective in the inhibition of abnormal production of reactive oxygen species (ROS) and reactive nitrogen species (RNS). The decelerated production of these reactive species can negatively affect normal cellular processes and physiological functions. ${ }^{7,8}$ The free radicals excess is associated with many chronic diseases development, including cancer, cardiovascular diseases, as well as various neurological and metabolic diseases. ${ }^{7,8}$ Making that vegetable origin substances with antioxidant properties become objects of several studies.

Despite the antioxidant activity presented by some essential oils, their applications may be hampered by their intrinsic characteristics, especially their high volatilities, instabilities, immiscibility and mainly solubility in aqueous media..$^{9,10}$ To overcome these disadvantages, a new approach has been proposed in which essential oils are

*e-mail: lguimaraes@ufsj.edu.br incorporated into nanogels, providing protection and preventing their degradation, as well as improving their stability and dispersion in aqueous solutions. ${ }^{11}$

Chitosan, with a molecular structure formed by N-acetyl-Dglucosamine and D-glucosamine $(\alpha-1,4)$, is a promising polymer for nanogels obtainment. The presence of the amine and hydroxyl groups gives this polymer viability for chemical modifications in its structure, since they allow the formation of covalent bonds with other species. ${ }^{12}$ By inserting other groups or molecules into the chitosan structure it is possible to obtain new materials in different forms and properties, including fibers, films, powders, capsules and gels. The main advantages of using chitosan are its easy obtainment, excellent biocompatibility and biodegradability. ${ }^{13}$

Some phenolic acids, including cinnamic, ferulic, p-coumaric and caffeic acids, have been used to promote chitosan structure modifications through crosslinking process, resulting in the nanogels production capable of encapsulating different essential oils. ${ }^{14,15}$ Zhaveh et al. ${ }^{16}$ reported the preparation of chitosan based on chitosan-caffeic acid nanogel by means of a reaction mediated by the 1-ethyl-3-(3-dimethylaminopropyl) carbodiimide (EDC) coupling agent. In this investigation, the promising characteristics of this new material were reported, including the increase in the antimicrobial performance of the Cuminum cyminum essential oil, used against the Aspergillus flavus fungus.

In this study, we evaluated a nanogel of modified chitosan (chitosan bound to caffeic acid) as an essential oil of L. origanoides encapsulating agent, in order to avoid its degradation and to improve its dispersion in aqueous solutions, reinforcing at the same time its antioxidant activity. The caffeic acid was selected as a natural phenolic compound, widely found in coffee, fruits, wine and olive oil, as well as having antioxidant properties, and good biocompatibility and biodegradability. ${ }^{17,18}$ Zhaveh et al.,${ }^{16}$ demonstrated that it is possible to obtain nanogels of chitosan bound to caffeic acid. However, in this study we present a new approach to preparing nanogels of chitosan bound to caffeic acid, based on its ability to incorporate 
the $L$. origanoides essential oil, increasing its performance as an antioxidant.

The objectives of this study were, therefore, (i) to perform the synthesis of nanogel of chitosan bound to caffeic acid; (ii) L. origanoides essential oil encapsulation; (ii) to estimate its encapsulation efficiency, and (iii) to evaluate the nanogel antioxidant activity before and after the incorporation of the L. origanoides essential oil by different tests.

\section{EXPERIMENTAL}

\section{Materials}

The materials used for this research were the low molecular weight chitosan ( $\mathrm{DD}=75-85 \%$ and $\left.\mathrm{M}_{\mathrm{v}}=50,000-190,000 \mathrm{Da}\right)$, caffeic acid $(98 \%)$ e $N$-(3-Dimethylaminopropyl)- $N$ '-ethylcarbodiimide hydrochloride (EDC), ABTS (> 98\%), DPPH (97\%), trolox $(97 \%)$ obtained by Sigma Aldrich. All chemicals used were of analytical grade.

\section{Methods}

\section{Extraction of the essential oil}

The fresh leaves of $L$. origanoides collected in the municipality of Itumirim / MG (21 ${ }^{\circ} 12$ ' $58^{\prime \prime} \mathrm{S}, 44^{\circ} 51$ ' 21 ' W, $\left.837 \mathrm{~m}\right)$ were used to extract the essential oil. In agreement with the proposed method, ${ }^{19}$ the hydrodistillation technique in a modified Clevenger apparatus was used for extraction. The hydrolate obtained after extraction was collected and centrifuged, which was collected and stored under refrigeration at $4{ }^{\circ} \mathrm{C}$.

\section{Analysis of the essential oil}

The constituents of the eessential oil of $L$. origanoides were analyzed by means of gas chromatography equipped with a flame ionization detector (GC-FID) and gas chromatography coupled with mass spectrometry (GC-MS).

The GC-MS analysis of the essential oil was performed with a chromatography Shimadzu CG-17A coupled to mass spectrometry QP 5000. The equipment equipped with a fused silica capillary column $(30 \mathrm{~m} \times 0.25 \mathrm{~mm}$, film thickness $0.25 \mu \mathrm{m})$. For the detection, impact energy of $70 \mathrm{eV}$ was used. Carrier gas was helium at a flow rate of $1 \mathrm{~mL} \mathrm{~min}^{-1}$. Injector temperature was set at $220{ }^{\circ} \mathrm{C}$. The column programmed with initial temperature of $40^{\circ} \mathrm{C}$, being added $3{ }^{\circ} \mathrm{C}$ every minute until reaching $240{ }^{\circ} \mathrm{C}$; initial column pressure of $100.2 \mathrm{KPa}$; split ratio 1:10. Diluted samples (1\% solution in hexane) of $1 \mu \mathrm{L}$. The compounds identification was based on comparison of their relative retention times with those of hydrocarbon mixture $\left(\mathrm{C}_{9} \mathrm{H}_{20} \ldots \ldots \ldots . . . \mathrm{C}_{26} \mathrm{H}_{54}\right)$ injection made under the same conditions as the essential oils, and by matching their arithmetic indexes with those obtained from databases of the Wiley library database 229 and published data libraries. ${ }^{20}$

The GC-FID analysis of the essential oil was performed using a gas chromatograph Shimadzu, model GC-2010, equipped with a RTX-5MS capillary column $(30 \mathrm{~m} \times 0.25 \mathrm{~mm}$ i.d., $0.25 \mu \mathrm{m}$ film thickness). Nitrogen was the carrier gas, at a flow rate of $1.18 \mathrm{~mL} \mathrm{~min}^{-1}$. The injector and detector temperatures were set at 220 and $300{ }^{\circ} \mathrm{C}$, respectively. The program used was $60^{\circ} \mathrm{C}$ rising to $240{ }^{\circ} \mathrm{C}$ at a rate of $3{ }^{\circ} \mathrm{C} \mathrm{min}^{-1}$, passing to a heating rate of $10{ }^{\circ} \mathrm{C} \mathrm{min}{ }^{-1}$ raised to $300^{\circ} \mathrm{C}$ and finally remaining at that temperature for $10 \mathrm{~min}$. Diluted samples (1/100, v/v, in hexane) of $1.0 \mu \mathrm{l}$ were injected, split ratio 1:50 and pressure in the column of $115 \mathrm{KPa}$.

Chitosan nanogel bound to caffeic acid synthesis

Chitosan nanogel bound to caffeic acid was prepared as reported previously by Chen et al. ${ }^{21}$ First, $1.20 \mathrm{~g}$ of chitosan was dissolved in $100 \mathrm{~mL}$ of aqueous acetic acid (1\% v/v) under magnetic stirring. Then, $85 \mathrm{~mL}$ of methanol was added, being the solution subsequently submitted ultrasonic bath at the frequency of $40 \mathrm{kHz}$, at room temperature. Subsequently, $1.10 \mathrm{~g}$ of caffeic acid and $1.68 \mathrm{~g}$ of EDC was mixed and dissolved in $20 \mathrm{~mL}$ of ethanol. After that, both solutions were mixed under $350 \mathrm{rpm}$ stirred, following the reaction for 24 hours. After this period, the nanogel was submitted ultrasonic bath at the frequency of $40 \mathrm{kHz}$ and the solution was adjusted to 8.5-9.0 using a solution of sodium hydroxide $\left(1 \mathrm{~mol} \mathrm{~L}^{-1}\right)$. The mixture was then centrifuged at $5100 \mathrm{rpm}$ for 15 minutes to recover the precipitated nanogel, and subjected to a series of washes with distilled water until neutral $\mathrm{pH}$. In the sequel, the nanogel was submitted to three washes with ethanol, the blend being centrifuged at $5100 \mathrm{rpm}$ for $15 \mathrm{~min}$. The obtained nanogel was placed for drying under vacuum at natural temperature in a desiccator containing silica gel.

\section{Lippia origanoides essential oil encapsulation}

The encapsulation of L. origanoides essential oil into (CS-CA) was performed using approximately $100 \mathrm{mg}$ of CS-CA dissolved in $10 \mathrm{~mL}$ of aqueous acetic acid solution $(\mathrm{pH}=3.5-4.0)$ under stirring. The phase dispersed was composed of L. origanoides essential oil in ethanol (1:1 mass/volume). Under $500 \mathrm{rpm}$ stirring, the essential oil solution was dripped into the nanogel solution. After, the resulting mixture was sonicated to $40 \mathrm{kHz}$ frequency, for $10 \mathrm{~min}$ at room temperature. Then, the nanogel containing the essential oil had the $\mathrm{pH}$ adjusted to 8.5-9.0 employing sodium hydroxide $\left(1 \mathrm{~mol} \mathrm{~L}^{-1}\right)$. Several washes with distilled water were performed in the precipitated material until neutral $\mathrm{pH}$, and centrifuged at $5100 \mathrm{rpm}$ for $15 \mathrm{~min}$. Lastly, the precipitated (CS-CA) was transferred to a Teflon plate and placed for drying under vacuum at natural temperature in a desiccator containing silica gel. They were stored at $4{ }^{\circ} \mathrm{C}$.

\section{Nanogel characterization by ATR-FTIR}

Characterizations of pure chitosan, caffeic acid and nanogel CS-CA were determined by ATR-FTIR. FTIR spectra were recorded with a PerkinElmer (Frontier Single Range-MIR) spectrometer, coupled with ATR module. The ATR-FTIR spectra of the samples in the spectral region $4000-650 \mathrm{~cm}^{-1}$, were obtained by accumulating 16 scans at $4 \mathrm{~cm}^{-1}$ resolution, using $\mathrm{LiTaO}_{3}$ detector.

\section{Nanogel characterization by SEM}

The morphology of the obtained dehydrated nanogel (CS-CA) and of dehydrated nanogel encapsulated with essential oil of L. origanoides (CS-CAEO) was observed with scanning electron microscopy SEM (LEO EVO 40 model). Some selected samples were immersed in liquid nitrogen and fractured on a cooled metal surface to internal structures visualization. Gold particles were deposited onto the powders with a Balzers SCD 050 evaporator. The morphological analyses were performed in 100x and 500x approximations, of the surface and fracture (cross section) of the material.

\section{Nanogel characterization by DLS}

The particle size of the nanogels CS-CA previously and after the incorporation of the essential oil of $L$. origanoides were measured by dynamic light scattering (DLS, Beckman Couter DelsaNano C). For the analysis, $30 \mathrm{mg}$ of each sample was dispersed in $3 \mathrm{~mL}$ of aqueous solution of acetic acid (1\% v/v) under magnetic stirring for $24 \mathrm{~h}$ and taken to an ultrasonic bath (Unique brand and model USC $1600 \mathrm{~A}$ ), at a frequency of $40 \mathrm{kHz}$, for $10 \mathrm{~min}$ at room temperature. The DLS of the samples was performed using a dispersion angle $(\theta)$ of $173-25^{\circ}$ and a pinhole $(50 \mu \mathrm{m})$. 
Nanogel characterization by TGA

Termogravimetry analysis (TGA) of samples was performed on Shimadzu DTG- $60 \mathrm{H}$ instrument. Samples were placed alumina and were subject to a heating rate of $10{ }^{\circ} \mathrm{C} \mathrm{min}-1$ from $25^{\circ} \mathrm{C}$ to $800{ }^{\circ} \mathrm{C}$, under a nitrogen atmosphere. Samples of chitosan, caffeic acid, (CS-CA) nanogel, nanogel containing the encapsulated essential oil the $L$. origanoides, and the essential oil were analyzed.

Nanogel Encapsulation Efficiency (EE) by Gravimetric Analysis

After the incorporation of the L. origanoides essential oil by the polymeric matrix the maximum essential oil volatilization temperature was determined, by means of TGA analysis. For this, $0.100 \mathrm{~g}$ of chitosan-caffeic acid nanogel encapsulated with essential oil (CS-CAEO). The samples were then placed in an oven at $220{ }^{\circ} \mathrm{C}$ for a period of 12 hours. After that period, the samples were weighed and the encapsulation efficiencies (EE) calculated in accordance with Equation 1.

$\mathrm{EE}=\frac{\text { mass of essential oil in nanogel }}{\text { initial essential oil mass }} \times 100$

The mass of essential oil in nanogel refers the obtained mass after the gravimetric treatment, and the initial essential oil mass refers the oil mass initially weighed for be used in the nanogel encapsulation.

\section{Determination of nanogel antioxidant activity}

Antioxidant activities of sample was investigated by using 1,1-diphenyl-2-picrylhydrazyl (DPPH) radical scavenging, ABTS radical scavenging assays and FRAP ferric ion-reducing, performed according to Teixeira et al. ${ }^{22}$

\section{DPPH assay}

For DPPH radical scavenging activity, $0.5 \mathrm{~mL}$ of samples (CS-CA) nanogel, nanogel containing the encapsulated essential oil the $L$. origanoides, and pure essential oil in concentration of $3 \mathrm{mg}$ $\mathrm{mL}^{-1}$ were added to a $2.5 \mathrm{~mL}$ of DPPH radical solution $(60 \mu \mathrm{M})$. The mixture was shaken vigorously and allowed standing for $30 \mathrm{~min}$. The DPPH solution without the materials was used as control. Absorbance of the resulting solution was measured at $515 \mathrm{~nm}$ by a spectrophotometer. The results obtained were expressed in two different ways, mg of nanogel/mg of DPPH and free radical neutralization percentage (\% NFR). The $\mathrm{mg}$ of nanogel $/ \mathrm{mg}$ of DPPH was determined from a DPPH analytical curve at concentrations of $10,20,30,40,50$ and $60 \mu \mathrm{M}$. On the other hand, (\% NFR) being calculated using Equation 2:

$\% \mathrm{NFR}=\frac{\text { control absorbance }- \text { sample absorbance }}{\text { control absorbance }}$

The control absorbance refers the DPPH solution absorbance without the materials, while and sample absorbance refers the absorbance is obtained for the nanogel after the analysis.

\section{ABTS assay}

For ABTS radical scavenging activity, the ABTS radical was prepared by reacting of $5 \mathrm{~mL}$ of the ABTS solution $(7 \mathrm{mM})$ with potassium persulfate solution $(140 \mathrm{mM}) 88 \mu \mathrm{L}$. The resulted solution kept at room temperature in the absence of light for 16 hours. After, the resulting solution was diluted with ethanol until display a $734 \mathrm{~nm}$ an absorbance of 0.700 .

For determine the activity of samples (CS-CA) nanogel, nanogel containing the encapsulated essential oil the L. origanoides, and pure essential oil) three solutions of each were prepared. The nanogel
(CS-CA) was prepared at the concentrations of 1,2 and $3 \mathrm{mg} \mathrm{mL}^{-}$ 1 , while the essential oil the concentrations were $0.10,0.25$ and $0.50 \mathrm{mg} \mathrm{mL}^{-1}$ in ethanol/water (1:1). Absorbance of the resulting solutions was measured at $734 \mathrm{~nm}$ by a spectrophotometer, being the Trolox used as reference $(50,100,500,1000,1500$ and 2000 $\mu \mathrm{M}$ in ethanol). For analysis, $30 \mu \mathrm{L}$ aliquot of each diluted nanogels solutions, and pure essential oil were added to a $3.0 \mathrm{~mL}$ of the ABTS radical solution and after 6 minutes absorbance was read. The results were expressed as $\mathrm{mg}$ of trolox/g nanogel.

\section{FRAP assay}

For determine the ferric reducing antioxidant power a stock solutions of $0.3 \mathrm{mM}$ acetate buffer, $10 \mathrm{mM}$ TPTZ (2, 4, 6-tripyridylStriazine in $40 \mathrm{mM} \mathrm{HCl}$ ) and $20 \mathrm{mM} \mathrm{FeCl}_{3} \cdot 6 \mathrm{H}_{2} \mathrm{O}$ were prepared. The working FRAP reagent was prepared by mixing these stock combination of $25 \mathrm{~mL}$ acetate buffer solution, $2.5 \mathrm{~mL}$ TPTZ solution, and $2.5 \mathrm{~mL}$ ferric chloride solution. Then, $90 \mu \mathrm{L}$ of samples solutions (chitosan $=2.5 \mathrm{mg} \mathrm{mL}^{-1}$, nanogel CS-CA $=3.75 \mathrm{mg} \mathrm{mL}^{-1}$ and nanogel $\mathrm{CS}-\mathrm{CAEO}=0.25 \mathrm{mg} \mathrm{mL}^{-1}$ ) was mixed with $2.7 \mathrm{~mL}$ of the working solution and $270 \mu \mathrm{L}$ of pure water, and the mixture was incubated at temperature of $37{ }^{\circ} \mathrm{C}$ for $30 \mathrm{~min}$ in the dark. Absorbance was measured at $595 \mathrm{~nm}$. The analyses were carried out utilizing different concentrations of $500,1000,1500$, and $2000 \mu \mathrm{M}$ ferrous sulfate is distilled water. The FRAP reagent was used as a blank. The FRAP value was expressed as $\mathrm{mM}$ ferrous sulfate per gram of samples.

\section{RESULTS AND DISCUSSION}

\section{Lippia origanoides essential oil characterization and identification}

The essential oil GC-MS analysis revealed the presence of nineteen compounds, representing $97.91 \%$ of the essential oil chemical composition (Table 1). As shown in this table, thymol $(78.20 \%), \rho$-cymene $(5.76 \%),(E)$-caryophyllene $(3.53 \%)$ and $\gamma$-terpinene $(3.33 \%)$ were the major L. origanoides essential oil components.

These results (Table 1) concerning the chemical composition of the $L$. origanoides essential oil corroborate with those obtained in other studies. Oliveira et al., ${ }^{23}$ analyzed the chemical composition of the $L$. origanoides essential oil and obtained thymol as the major compound, followed by $\rho$-cymene and $(E)$-caryophyllene. Recent studies by Damasceno et al., ${ }^{6}$ evaluating the essential oil extracted from this plant, also described as major component thymol (78.20\%), followed by $\rho$-cymene $(5.76 \%)$ and $(E)$-caryophyllene $(3.53 \%)$. However, the chemical composition of the essential oils is dependent on the plant physiology, so the L. origanoides essential oils may present as major constituents other compounds, such as carvacrol, $\rho$-cymene, $\alpha$ - and $\beta$-phellandrene, limonene and 1,8-cineol. ${ }^{24,25}$

\section{Nanogel characterization by ATR-FTIR}

The result of the synthesis of chitosan-caffeic acid was evaluated based on the ATR-FTIR spectra obtained (Figure 1).

The ATR-FTIR identification of the binding between CS and CA (Figure 1B) was observed by the presence of the absorption band at $1552 \mathrm{~cm}^{-1}$ regarding the $\mathrm{C}=\mathrm{C}$ stretching of aromatic ring. In addition, it was possible to observe the absence of the $\mathrm{N}-\mathrm{H}$ deformation vibrations of chitosan amide II at $1586 \mathrm{~cm}^{-1}$. It was also possible to observe in the spectrum of CS-CA nanogel the reduction in the intensity of the absorption band in the region of $3326 \mathrm{~cm}^{-1}$, which corresponds the primary amine groups, while secondary amides were formed. In addition, it was possible to observe the broadening and 
Table 1. Chemical composition of the L. origanoides essential oil obtained by GC-MS and GC-FID analysis

\begin{tabular}{|c|c|c|c|c|}
\hline & Compounds & $\mathrm{CAI}^{\mathrm{a}}$ & $\mathrm{TAI}^{\mathrm{a}}$ & $\%^{a}$ \\
\hline 1 & $\alpha$-thujene & 920 & 924 & 0.53 \\
\hline 2 & $\alpha$-pinene & 933 & 932 & 0.38 \\
\hline 3 & $\beta$-pinene & 978 & 974 & 0.85 \\
\hline 4 & Myrcene & 983 & 988 & 0.18 \\
\hline 5 & $\alpha$-phellandrene & 1006 & 1002 & 0.09 \\
\hline 6 & $\alpha$-terpinene & 1018 & 1014 & 1.12 \\
\hline 7 & $\rho$-cymene & 1025 & 1020 & 4,89 \\
\hline 8 & Limonene & 1024 & 1024 & 1.13 \\
\hline 9 & 1,8 -cineole & 1026 & 1026 & 1.36 \\
\hline 10 & $\gamma$-terpinene & 1054 & 1054 & 0.98 \\
\hline 11 & cis-hydrated sabinene & 1065 & 1065 & 0.25 \\
\hline 12 & Terpinolene & 1090 & 1086 & 0.42 \\
\hline 13 & trans-hydrate sabinene & 1102 & 1098 & 0.15 \\
\hline 14 & $\alpha$-terpineol & 1194 & 1186 & 1.13 \\
\hline 15 & Thymol methyl ether & 1230 & 1232 & 0.74 \\
\hline 16 & Thymol & 1289 & 1289 & 76.90 \\
\hline 17 & Thymol acetate & 1364 & 1355 & 0.33 \\
\hline 18 & (E)-caryophyllene & 1417 & 1417 & 4.92 \\
\hline 19 & $\alpha$-humulene & 1447 & 1452 & 0.10 \\
\hline \multicolumn{4}{|c|}{ Monoterpene hydrocarbons } & 10.57 \\
\hline \multicolumn{4}{|c|}{ Oxygenated monoterpenes } & 80.86 \\
\hline \multicolumn{4}{|c|}{ Sesquiterpene hydrocarbons } & 0.10 \\
\hline \multicolumn{4}{|c|}{ Oxygenated sesquiterpenes } & 4.92 \\
\hline \multicolumn{4}{|c|}{ Total } & 96.45 \\
\hline
\end{tabular}

$\overline{{ }^{a} \text { CAI - calculated arithmetic index, TAI - tabulated arithmetic index and }}$ (\%) - concentration ${ }^{20}$.

increasing of intensity of the absorption band at $1643 \mathrm{~cm}^{-1}$, which corresponds to the $\mathrm{C}=\mathrm{O}$ stretching vibrations of amide $\mathrm{I}$. Results of the ATR-FTIR spectra of chitosan is shown in Figures 1A-B and described in Table 2. ${ }^{26,27,16}$

The morphology of dehydrated nanogels was analyzed by MEV to estimate the effect of the L. origanoides essential oil on the CS-CA matrix structure. Figure 2 shows the morphology of the obtained CS-CA nanogel.

After drying CS-CA nanogel, it was not possible to observe the formation of well-defined particles (Figure 2), but an irregular agglomeration with different sizes and shapes. At basic $\mathrm{pH}$ the free amino groups of chitosan are deprotonated which causes the reduction of the electrostatic repulsion between their particles. As a result, nanogel when precipitated tends to rapidly aggregate its particles irregularly. ${ }^{28}$

Electromicrographs of the cross-sections and surfaces of CS-CA nanogels incorporated with $L$. origanoides essential oil are shown in Figure 3. After the incorporation of the essential oil into the polymer matrix, changes in the structure of the material are observed by the electromicrographs (Figure 3).

It is possible to observe on the nanogel surface (Figure $3 \mathrm{~A}$ ) the presence of irregular regions and well defined spherical cavities, distributed throughout the material. However, these characteristics were not observed in the control material (Figure 2), which presented an uniform external structure, without cracking or pores, indicating the formation of a dense and compact structure.
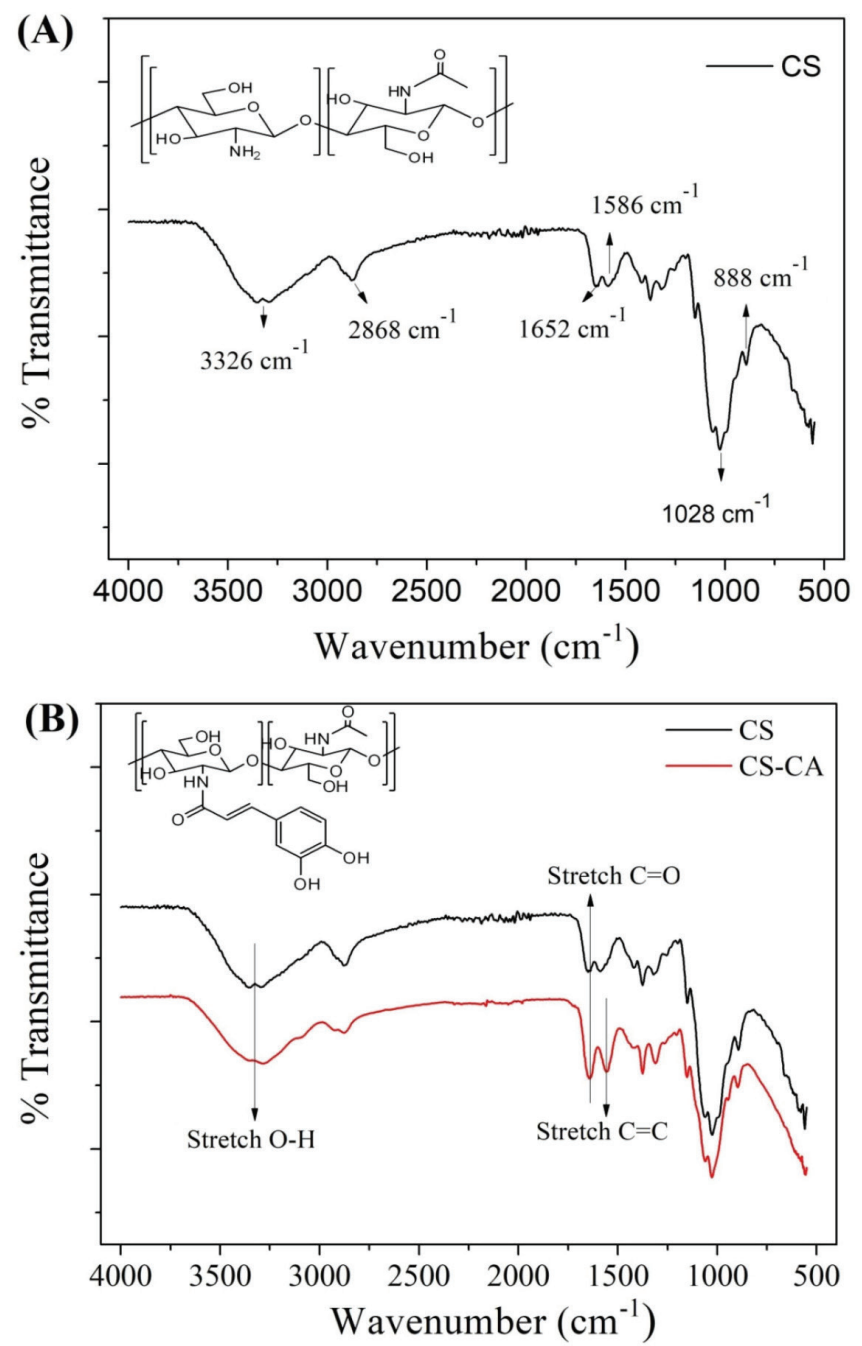

Figure 1. FTIR spectra of $(A)$ chitosan and (B) chitosan-caffeic acid nanogel and chitosan

Table 2. Bands observed in the ATR-FTIR spectra for chitosan with their attributions

\begin{tabular}{cc}
\hline \multicolumn{3}{c}{ Chitosan } \\
\hline IR $\left(\mathrm{cm}^{-1}\right)$ & Attribution \\
\hline 3326 & $v(\mathrm{O}-\mathrm{H})$ and $v(\mathrm{~N}-\mathrm{H})$ \\
2868 & $v(\mathrm{C}-\mathrm{H})$ \\
1643 & $v(\mathrm{C}=\mathrm{O})$ amide \\
1586 & $\delta(\mathrm{N}-\mathrm{H})$ amide \\
1028 & $v(\mathrm{C}-\mathrm{O}-\mathrm{C})$ glycosidic link \\
888 & $v(\mathrm{C}-\mathrm{O})$ alcohols \\
\hline
\end{tabular}

On Figure 3B it is possible to see the presence of a larger number of pores and spherical cavities in the sample transversal section, which are well dispersed into the CS-CA matrix. These results indicate that the oil droplets were adhered effectively within the CS-CA matrix. Almeida et al. ${ }^{15}$ reported similar results when the $L$. origanoides essential oil was incorporated in nanogels of chitosan-ferulic acid.

\section{Determination of Particle Size and Zeta Potential from nanogels}

The dynamic light scattering (DLS) technique was used to 


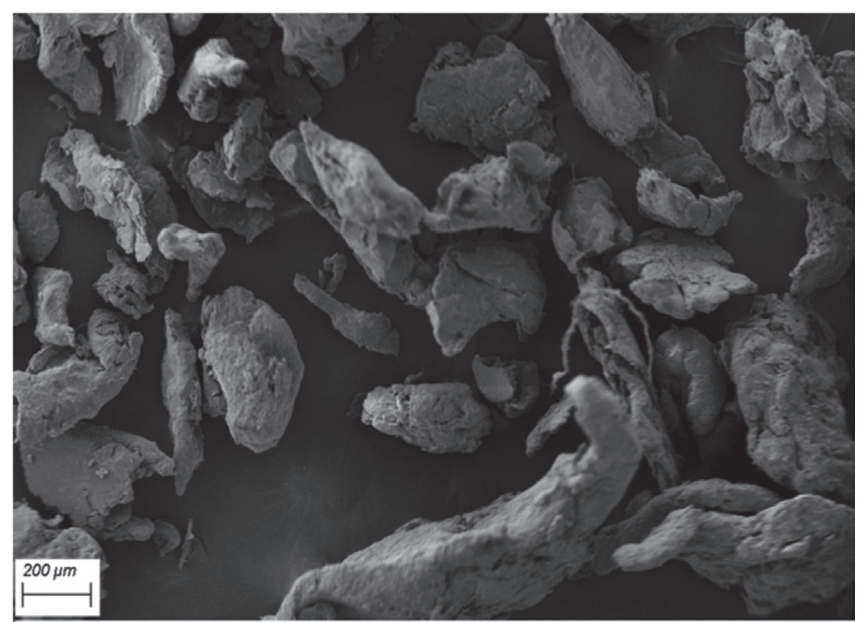

Figure 2. Electromicrograph of chitosan-caffeic acid nanogel (approximation $100 x)$

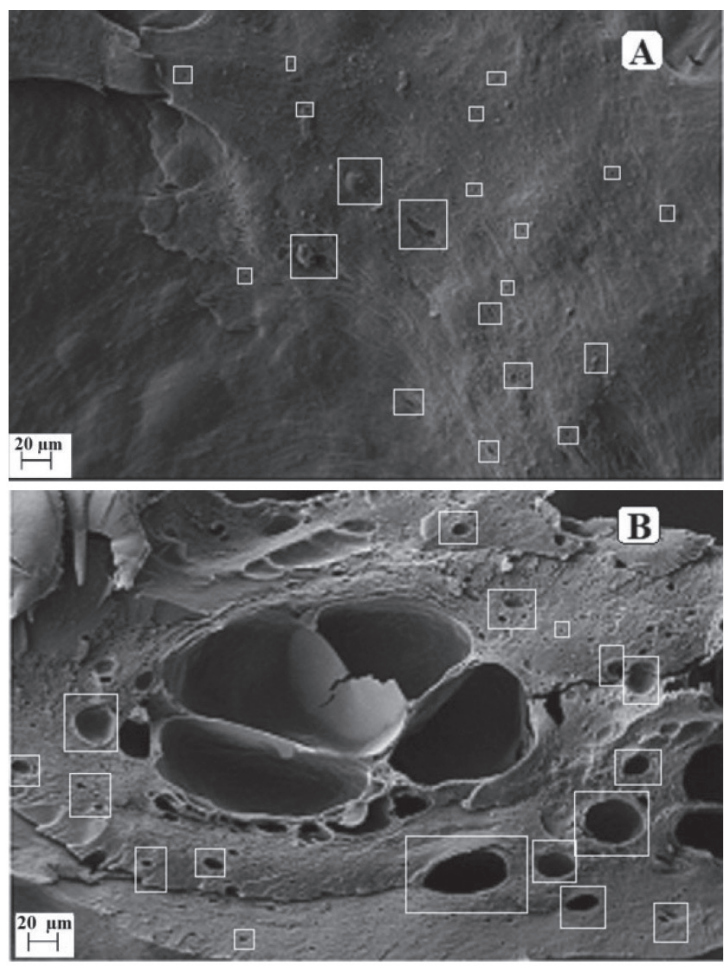

Figure 3. Electromicrographs: A) surface of chitosan-caffeic acid nanogel (approximation 500x); B) cross section of chitosan-caffeic acid nanogel (approximation 500x)

determine the mean particle size of CS-CA nanogel and its Zeta potential before and after the incorporation of the essential oil of L. origanoides. Table 3 presents the results obtained.

It can be observed from the Table 3 that with the encapsulation of the essential oil of $L$. origanoides by the nanogels a larger average particle size was obtained. The data indicate that nanogel-essential

Table 3. Medium size values and nanogels zeta potential of nanogels

\begin{tabular}{ccc}
\hline Sample $^{\text {a }}$ & $\begin{array}{c}\text { Mean size particle } \\
\text { size }(\mathrm{nm})\end{array}$ & Zeta potencial $(\mathrm{mV})$ \\
\hline CS-CA & $161.75 \pm 70.56 \mathrm{~nm}$ & +49.61 \\
CS-CAEO & $417.26 \pm 55.96$ & +54.57 \\
\hline
\end{tabular}

${ }^{a}$ CS-CA - chitosan-caffeic acid nanogel and CS-CAEO - chitosan-caffeic acid nanogel containing encapsulated essential oil. oil interactions significantly affect the structure of nanogel. Hasheminejad et al. ${ }^{29}$ also observed in their studies that after the incorporation of the clove essential oil by the chitosan polymer matrix, the particle size increased significantly.

The results of zeta potential Table 3 indicate that similarly to the nanogel of CS-CA, the nanogel of CS-CAEO presents amine groups protonated on its surface and, consequently, has a positive surface charge. Moreover, by the results it is possible to observe that when incorporating the essential oil of $L$. origanoides the particles of nanogel became more stable, that is, they presented higher values of zeta potentials. Despite the increase in particle stability upon incorporation of the essential oil, the result obtained for the CS-CA sample also indicates good stability. According to Zhao et al..$^{30}$ materials exhibiting zeta potential values in module, above $30 \mathrm{mV}$ are more stable. This is due to the fact that repulsive forces act on the particles of the material, thus preventing their aggregation.

\section{Thermal analysis}

The synthesized CS-CA nanogel thermal decomposition was investigated by thermogravimetric analysis (TGA). On Figures 4A-B the control TGA curves (chitosan and caffeic acid) are presented. The chitosan presents three characteristic thermal events, while the caffeic acid two events related to its decomposition.

All the thermal event of mass loss of chitosan and caffeic acid shown in Figures 4A-B are described in Table 4..$^{15,6}$

The thermogravimetric curve of the nanogel of chitosan-caffeic acid presented three thermal decomposition events (Figure 4C and Table 4), similar to chitosan. However, the second thermal event (232-414 ${ }^{\circ} \mathrm{C}$ ) for nanogel degradation was slower compared to pure chitosan $\left(245-378^{\circ} \mathrm{C}\right)$. In this way, the modified polymer presented greater thermal stability in relation to chitosan. This result confirms the successful formation of CS-CA binding since chitosan modified with caffeic acid may have undergone modifications in its intramolecular and intermolecular bonds in order to influence its stability. ${ }^{31}$

After the incorporation of the essential oil by the polymer matrix, a new mass loss event, related to the volatilization of the essential oil, is observed. The mass loss processes related to the volatilization of the essential oil shown in Figure 4D are described in Table 5.

For all the CS-CAEO samples, it was verified that the temperature that starts the volatilization of the essential oil $\left(\mathrm{T}_{\text {onset }}\right)$ and ends $\left(\mathrm{T}_{\text {endset }}\right)$, occurred at higher temperatures than in the pure essential oil (Table 5). Inferring that the interaction between the nanogel and the essential oil made it difficult to volatilize. These results confirm those obtained by MEV, that most of the L. origanoides essential oil is stably incorporated into the CS-CA nanogel.

\section{Encapsulation efficiency (EE) by Gravimetric Analysis}

After the TGA analysis (Figure 4), it was possible to determine the volatilization temperature of the L. origanoides essential oil, which occurred between $22-255^{\circ} \mathrm{C}$, when incorporated to the CS-CA nanogel. By means of these results, the temperature used in the gravimetric analysis was determined. The temperature of $220{ }^{\circ} \mathrm{C}$ was selected, since at this temperature only the essential oil would be volatilized, and no events related to CS-CA nanogel degradation were observed (Tables 3 and 4). Thus, the values related to the encapsulation efficiencies of the CS-CA nanogel (EE) were calculated according to Equation 1. The CS-CA nanogel exhibited an average encapsulation efficiency of $45 \pm 2 \%$.

With base on the encapsulation efficiencies of $L$. origanoides essential oil obtained by other matrices in recent works, it is observed that the obtained result in this work was satisfactory. 

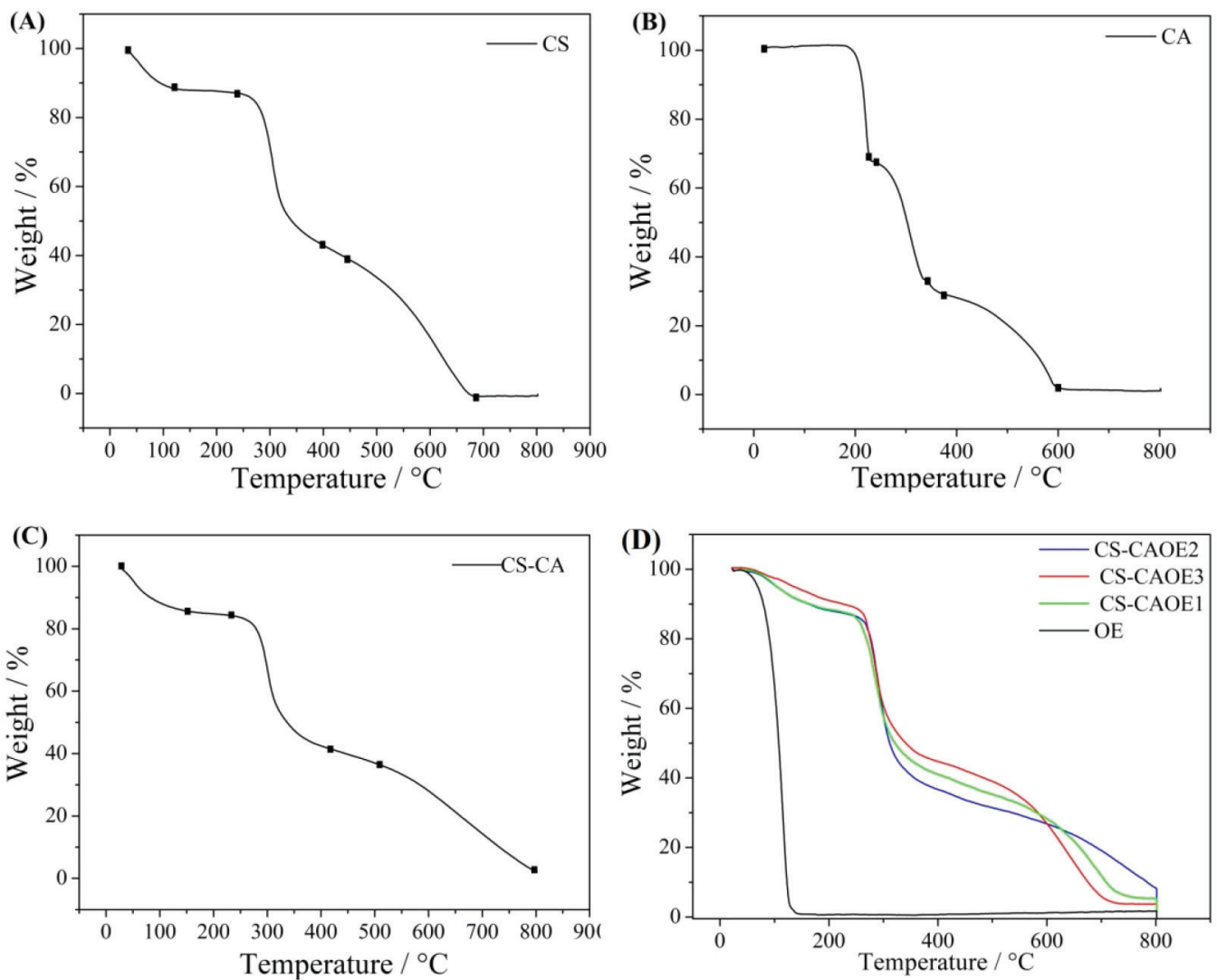

Figure 4. TGA curves of chitosan (A), caffeic acid (B), chitosan-caffeic acid nanogel (C) and chitosan-caffeic acid nanogel containing encapsulated essential oil (D)

Table 4. TGA data for chitosan (CS), caffeic acid (FA) and CS-CA nanogels

\begin{tabular}{|c|c|c|c|c|c|}
\hline Sample & Event & $\mathrm{T}_{\text {onset }}\left({ }^{\circ} \mathrm{C}\right)$ & $\mathrm{T}_{\text {endset }}\left({ }^{\circ} \mathrm{C}\right)$ & Mass Loss $(\%)$ & Explanation \\
\hline \multirow{3}{*}{ CS } & 1 & 35 & 124 & 10.91 & Loss of water molecules \\
\hline & 2 & 239 & 387 & 44.02 & Polymer decomposition \\
\hline & 3 & 447 & 687 & 39.99 & Polymer degradation and loss of inorganic material \\
\hline \multirow{3}{*}{$\mathrm{CA}$} & 1 & 172 & 222 & 4.0 & Compound decomposition \\
\hline & 2 & 242 & 345 & 64.0 & Compound decomposition \\
\hline & 3 & 370 & 596 & 30.0 & Compound decomposition \\
\hline \multirow{3}{*}{ CS-CA } & 1 & 33 & 146 & 14.6 & Loss of water molecules \\
\hline & 2 & 232 & 414 & 42.6 & Polymer degradation \\
\hline & 3 & 511 & 800 & 34.16 & Decomposition of nanogel \\
\hline
\end{tabular}

Table 5. TGA data for CS-CA nanogels containing essential oil

\begin{tabular}{cccc}
\hline Sample $^{\mathrm{a}}$ & $\mathrm{T}_{\text {onset }}\left({ }^{\circ} \mathrm{C}\right)$ & $\mathrm{T}_{\text {endset }}\left({ }^{\circ} \mathrm{C}\right)$ & Mass Loss $(\%)$ \\
\hline CS-CAEO1 & 22 & 248 & 11.18 \\
CS-CAEO2 & 36 & 255 & 13.06 \\
CS-CAEO3 & 34 & 240 & 12.74 \\
EO Lippia origanoides & 21 & 123 & 98.61 \\
\hline
\end{tabular}

${ }^{a}$ CS-CAEO - chitosan-caffeic acid nanogel containing encapsulated essential oil, EO1, EO2 e EO3 - refers to triplicates.

Damasceno et al. ${ }^{6}$ evaluated the efficiency of pure chitosan and $\rho$-coumaric chitosan-acid nanogel by gravimetric analysis to encapsulate the $L$. origanoides essential oil, obtaining results of $37 \pm 2$ and $42 \pm 1$, respectively. Whereas, Almeida et al. ${ }^{15}$ using the thermogravimetric analysis evaluated the ability of the nanogel of chitosan-ferulic acid to encapsulate the $L$. origanoides essential oil. The authors obtained 20\% EE in the proportion of (3.9: 9.9) of (ferulic acid: EDC), $12.0 \%$ (2.0: 5.0), $10.0 \%(1.0: 2.5)$ and $13.4 \%(0.5: 1.3) .{ }^{15}$ These contents are inferior to the one found in this study of $45 \pm 2$, which shows the potential of the CS-CA nanogel to satisfactorily encapsulate the L. origanoides essential oil.

\section{Antioxidant activity by DPPH assay}

The assay based on neutralization of the DPPH radical has been widely used to determine the ability of neutralizing free radicals. ${ }^{32}$ In reaction processing, antioxidants react with $\mathrm{DPPH}$, reducing a number of DPPH molecules, equal to the number of hydrogen radicals that may be available. In the neutralization process of the DPPH free radical it is possible to visualize a discoloration of the solution from purple to yellow. ${ }^{33}$ 
The antioxidant activities found for L. origanoides EO and CS-CA nanogel with and without incorporated essential oil are shown in Table 6. The results were expressed in mg sample/mg DPPH and also in $\%$ neutralization of the free radicals.

It can be observed from the results (Table 6) that the CS-CA nanogel already had an antioxidant activity, probably due to the presence of the caffeic acid in the chitosan structure. Aytekin et al. ${ }^{34}$ showed that the highest antioxidant activity occurred in the chitosan derivatives containing the highest proportion of caffeic acid, confirming that the antioxidant capacity of chitosan was enhanced by its conjugation with the acid.

Table 6. Antioxidant activity by DPPH radical neutralization

\begin{tabular}{ccc}
\hline Samples $^{\text {a }}$ & $\begin{array}{c}\text { mg of sample/mg of } \\
\text { DPPH }\end{array}$ & $\begin{array}{c}\text { \% of free radical } \\
\text { neutralization }\end{array}$ \\
\hline CS-CA & 53.47 & $25.16 \pm 1.75$ \\
CS-CAEO & 34.48 & $61.81 \pm 0.69$ \\
EO & 27.34 & $78.09 \pm 3.28$ \\
\hline
\end{tabular}

${ }^{a}$ CS-CA - chitosan-caffeic acid nanogel, CS-CAEO - chitosan-caffeic acid nanogel containing encapsulated essential oil and EO - essential oil.

Damasceno et ll $^{6}$ in a similar study with the modification of chitosan with p-coumaric acid, obtained by the DPPH test a lower antioxidant activity of $4.34 \pm 0.20 \%$, in comparison to the obtained result in this work (Table 6). Confirming the satisfactory antioxidant capacity of the modified chitosan with the caffeic acid.

After the incorporation of the essential oil in the CS-CA nanogel it is observed that there was a significant increase in the \% of neutralization of the free radicals comparing with the material without the presence of oil. It is important to note that the essential oil encapsulation in the CS-CA nanogel showed the best result, since the $\%$ neutralization values of the free radicals were close to those found for the pure L. origanoides essential oil (Table 6).

The relevant antioxidant activity presented by EO L. origanoides is mainly due to its main constituents. The constituents such as thymol and carvacrol are recognized as excellent bioactive molecules, being able to reduce the formation of free radicals. ${ }^{35}$

In this way, it can be observed that the increase in the antioxidant activity of the materials is related to the incorporation of the essential oil by the synthesized materials, as well as the formation of chemical bond between the chitosan and the caffeic acid, since this compound also presents activity.

\section{Antioxidant activity by ABTS assay}

The ABTS radical scavenging assay has also been widely used to evaluate antioxidants. In this assay, the ABTS moiety measures the neutralizing ability of free radicals by discoloring the ABTS blue reagent. The decrease in ABTS concentration is linearly dependent on the antioxidant concentration, including trolox as a reference antioxidant. ${ }^{36}$

The antioxidant activities found for EO L. origanoides and CS-CA nanogel with and without incorporated essential oil are shown in Table 7. The results were expressed as mg trolox/g sample.

By Table 7 it is possible to see that after the essential oil incorporation into the CS-CA nanogel there was a significant increase in the antioxidant activity. The value related to the antioxidant activity of the CS-CA nanogel (Table 7) was lower than that presented by the CS-CAEO nanogel after the incorporation of the essential oil.

By means of the obtained results, it is observed that after the encapsulation of the L. origanoides essential oil by the material, there
Table 7. Antioxidant activity by ABTS radical neutralization

\begin{tabular}{cc}
\hline Samples $^{\text {a }}$ & mg of trolox/g of sample \\
\hline CS-CA & $41.5 \pm 2.3$ \\
CS-CAEO & $396.2 \pm 10.7$ \\
EO & $365.9 \pm 12.1$ \\
\hline
\end{tabular}

${ }^{a}$ CS-CA - chitosan-caffeic acid nanogel, CS-CAEO - chitosan-caffeic acid nanogel containing encapsulated essential oil and EO - essential oil.

was a significant increase of the antioxidant activity of the resulting material. Being important to emphasize that for the CS-CAEO nanogel, the value was even higher than that presented by pure essential oil (Table 7).

\section{Antioxidant activity by FRAP assay}

Some compounds are inactive in the DPPH and ABTS assay but exhibit high antioxidant activities in the FRAP assay. Thus, the evaluation of these samples for their antioxidant behavior by the FRAP assay is of significant importance. The evaluation of antioxidants by this method is based on the reduction of the complex $\left(\mathrm{Fe}^{3+}-\mathrm{TPTZ}\right)$ by antioxidants to the ferrous form (Fe $\left.{ }^{2+}-\mathrm{TPTZ}\right){ }^{37}$

The Table 8 shows that after the incorporation of the essential oil into the CS-CA nanogel there was a substantial improvement in its antioxidant capacity. On the other hand, it is observed that by encapsulating the components present in the essential oil by means of the interactions with the polymer (Table 8), it reduced its availability for iron reduction by giving a low value relative to the pure L. origanoides essential oil.

Table 8. Antioxidant activity by FRAP reduction of the complex

\begin{tabular}{cc}
\hline Samples $^{\text {a }}$ & $\mu \mathrm{M}$ of ferrous sulphate/g of sample \\
\hline CS-CA & $57.76 \pm 8.86$ \\
CS-CAEO & $255.49 \pm 17.69$ \\
EO & $68865.11 \pm 321.40$ \\
\hline
\end{tabular}

${ }^{a}$ CS-CA - chitosan-caffeic acid nanogel, CS-CAEO - chitosan-caffeic acid nanogel containing encapsulated essential oil and EO - essential oil.

The antioxidant activity of the CS-CAEO nanogels by the methods studied in this work followed the order: ABTS $>$ FRAP $>$ DPPH. The results (Table 6, 7 and 8) indicated that during the encapsulation process potential antioxidant compounds present in the $L$. origanoides essential oil were effectively encapsulated. As a result was observed an increase in the antioxidant capacity of nanogel CS-CA under different assays.

\section{CONCLUSIONS}

The CS-CA nanogel was synthesized by the formation of the chitosan-caffeic acid bond. The interaction between CA and CS was confirmed by spectroscopy (ATR-FTIR) and thermochemical analysis (TGA). Through the gravimetric analysis it was verified that the synthesized nanogel presents an efficacy in the L. origanoides essential oil encapsulation, that among its main constituents, compounds such as thymol and $\rho$-cymene are present. This result was confirmed by morphological analysis, since after the incorporation of the essential oil by the CS-CA material, there were changes in its structure. On the surface and cross-section of the CS-CAEO sample the presence of irregular regions and well-defined spherical cavities was observed, which was not observed in the control sample. These 
results indicated that the droplets of essential oil were adhered effectively inside the matrix. The size distribution analysis showed that only with the incorporation of the essential oil is it possible to carry out the measurement of the CS-CA particle size. In relation to the antioxidant activities, it was observed that these were superior after the incorporation of the essential oil by the nanogels. It is important to note that for the nanogel of CS-CAEO, the value was even higher than that presented by pure essential oil.

\section{ACKNOWLEDGMENT}

To the Laboratory of Electron Microscopy and Ultrastructural Analysis (LME), at the Federal University of Lavras, Lavras (UFLA) Minas Gerais State, Brazil. This work was supported by the "Conselho Nacional de Desenvolvimento Científico e Tecnológico" (CNPq), the Fundação de Amaparo à Pesquisa do Estado de Minas Gerais" (FAPEMIG) and the "Coordenação de Aperfeiçoamento de Pessoal de Nível Superior” (CAPES).

\section{REFERENCES}

1. Nybo, S. E.; Kharel, M. K. In Studies in Natural Products Chemistry; Rahman, A. U., ed.; Elsevier: Cambridge, 2019, cap. 4.

2. Grande-Tovar, C. D.; Chaves-Lopez, C.; Serio, A.; Rossi, C.; Paparella, A.; Trends Food Sci. Technol. 2018, 78, 61

3. Bistgani, Z. E.; Siadat, S. A.; Bakhshandeh, A.; Pirbalouti, A. G.; Hashemi, M.; Maggi, F.; Morshedloo, M. R.; Ind. Crops Prod. 2018, 121, 434.

4. Mehdizadeh, L.; Moghaddam, M.; In Therapeutic, Probiotic, and Unconventional Foods; Grumezescu, A. M.; Holban, A. M., eds.; Elsevier: Cambridge, 2018, cap. 10.

5. Mar, J. M.; Silva, L. S.; Azevedo, S. G.; França, L. P.; Goes, A. F. F.; dos Santos, A. L.; de A. Bezerra, J.; de Cássia S.; Nunomura, R.; Machado, M. B.; Sanches, E. A.; Ind. Crops Prod. 2018, 111, 292.

6. Damasceno, E. T. S.; Almeida, R. R.; de Carvalho, S. Y. B.; de Carvalho, G. S. G.; Mano, V.; Pereira, A. C.; Guimarães, L. G. L.; Ind. Crops Prod. 2018, 125, 85.

7. Seifried, R. M.; Harrison, E.; Seifried, H. E.; In Nutrition in the Prevention and Treatment of Disease; Coulston, A. M.; Ferruzzi, M. G.; Boushey, C. J.; Delahanty, L. M., eds.; Elsevier: Cambridge, 2017, cap. 16.

8. Chedea, V. S.; Pop, R. M.; In Polyphenols in Plants; Watson, R. R., ed.; $2^{\text {nd }}$ ed., Elsevier: Cambridge, 2019, cap. 11.

9. Asbahani, A. E.; Miladi, K.; Badri, W.; Sala, M.; Addi, E. H. A.; Casabianca, H.; Mousadik, A. E.; Hartmann, D.; Jilale, A.; Renaud, F. N. R.; Elaissari, A.; Int. J. Pharm. 2015, 483, 220.

10. Navarro, R.; Arancibia, C.; Herrera, M. L.; Matiacevich, S.; Food Bioprod. Process. 2016, 97, 63.

11. Luckanagul, J. A.; Pitakchatwong, C.; Bhuket, P. R.; Muangnoi, C.; Rojsitthisak, P.; Chirachanchai, S.; Wang, Q.; Rojsitthisak, P.; Carbohydr. Polym. 2018, 181, 1119.
12. Croisier, F.; Jérôme, C.; Eur. Polym. J. 2013, 49, 780.

13. Oryan, A.; Sahvieh, S.; Int. J. Biol. Macromol. 2017, 104, 1003.

14. Beyki, M.; Zhaveh, S.; Khalili, S. T.; Rahmani-Cherati, T.; Abollahi, A.; Bayat, M.; Tabatabaei, M.; Mohsenifar, A.; Ind. Crops Prod. 2014, 54, 310.

15. Almeida, R. R.; Damasceno, E. T. S.; de Carvalho, S. Y. B.; de Carvalho, G. S. G.; Gontijo, L. A. P.; Guimarães, L. G. L.; Carbohydr. Polym. 2018, 188, 268.

16. Zhaveh, S.; Mohsenifar, A.; Beiki, M.; Khalili, S. T.; Abdollahi, A.; Rahmani-Cherati, T.; Tabatabaei, M.; Ind. Crops Prod. 2015, 69, 251.

17. Zhou, Q.; Yan, B.; Xing, T.; Chen, G.; Carbohydr. Polym. 2019, 203, 1.

18. Bai, R.; Yu, Y.; Wang, Q.; Fan, X.; Wang, P.; Yuan, J.; Shen, J. J.; Clean. Prod. 2018, 191, 48.

19. Agência Nacional de Vigilância sanitária; Farm. Bras. 2010, 1, 198.

20. Adams, R. P.; Identification of Essential Oil Components by Gas Chromatography, $4^{\text {th }}$ ed., Allured publishing: Illinois, 2017.

21. Chen, X. G.; Lee, C. M.; Park, H. J. J.; Agric. Food Chem. 2003, 51, 3135 .

22. Teixeira, T. S.; do Vale, R. C.; Almeida, R. R.; Ferreira, T. P. S.; Guimarães, L. G. L.; Rev. Virtual Química 2017, 9, 1546.

23. Oliveira, A. P.; Santos, A. A.; Santana, A. S.; Lima, A. P. S.; Melo, C. R.; Santana, E. D. R.; Sampaio, T. S.; Blank, A. F.; Araújo, A. P. A.; Cristaldo, P. F.; Bacci, L.; Crop Prot. 2018, 112, 33.

24. Sarrazin, S. L. F.; da Silva, L. A.; Oliveira, R. B.; Raposo, J. D. A.; da Silva, J. K. R.; Salimena, F. R. G.; Maia, J. G. S.; Mourão, R. H. V.; Lipids Health Dis. 2015, 14, 145 .

25. Ribeiro, A. F.; Andrade, E. H. A.; Salimena, F. R. G.; Maia, J. G. S.; Biochem. Syst. Ecol. 2014, 55, 249.

26. Liang, Y.; Zhao, X.; Ma, P. X.; Guo, B.; Du, Y.; Han, X.; J. Colloid Interface Sci. 2019, 536, 224.

27. Woranuch, S.; Yoksan, R.; Carbohydr. Polym. 2013, 96, 495.

28. Huang, Y.; Cai, Y.; Lapitsky, Y. J.; Mater. Chem. B 2015, 3, 5957.

29. Hasheminejad, N.; Khodaiyan, F.; Safari, M.; Food Chem. 2019, 275, 113.

30. Wang, L.; Chen, C.; In Toxicology of Nanomaterials; Zhao, Y.; Zhang, Z.; Feng, W., eds.; Wiley: Weinheim, 2016, cap. 11.

31. Szymańska, E.; Winnicka, K.; Mar. Drugs 2015, 13, 1819.

32. Hatamnia, A. A.; Abbaspour, N.; Darvishzadeh, R.; Food Chem. 2014, 145, 306.

33. Roby, M. H. H.; Sarhan, M. A.; Selim, K. A. H.; Khalel, K. I.; Ind. Crops Prod. 2013, 43, 827.

34. Aytekin, A. O.; Morimura, S.; Kida, K. J.; Biosci. Bioeng. 2011, 111, 212.

35. Teles, S.; Pereira, J. A.; Oliveira, L. M. de; Malheiro, R.; Machado, S. S.; Lucchese, A. M.; Silva, F.; Ind. Crops Prod. 2014, 59, 169.

36. Lupidi, G.; Maggi, F.; Morshedloo, M. R.; Quassinti, L.; Bramucci, M.; Nat. Prod. Res. 2017, 31, 2857.

37. Rui, L.; Xie, M.; Hu, B.; Zhou, L.; Saeeduddin, M.; Zeng, X.; Carbohydr. Polym. 2017, 170, 206. 